\title{
Les possibilités de sélection pour le rendement en grains du tournesol par l'étude de caractères morphologiques et architecturaux
}

\author{
Felicity VEAR ${ }^{1}$ \\ Anne-Marie TRIBOI ${ }^{1}$ \\ Jérémie LECOEUR ${ }^{2}$ \\ Patrick VINCOURT ${ }^{3}$ \\ Philippe DEBAEKE ${ }^{4}$ \\ 1 INRA, UMR 1095,
234 avenue du Brezet,
63100 Clermont-Ferrand, France
<vear@clermont.inra.fr>
${ }^{2}$ SUPAGRO, UMR LEPSE, 2, place Viala,
34060 Montpellier cedex 01, France
${ }^{3}$ INRA, UMR LIPM 441, BP 52627,
31326 Castanet-Tolosan, France
${ }^{4}$ INRA, UMR AGIR, BP 52627,
31326 Castanet-Tolosan cedex, France
}

Le rendement d'une culture est un caractère complexe, faiblement héritable et dont la mesure coûte cher. Pour le tournesol, il faut fabriquer des hybrides et les expérimenter dans plusieurs lieux. Ces dernières années, les évolutions des politiques agricoles ont amenéà cultiver le tournesol dans les moins bonnes terres avec pour conséquence une stagnation des rendements moyens nationaux. Des programmes impliquant l'ensemble de la profession (PROMOSOL : INRA, CETIOM, UFS) ont été entrepris pour faciliter une sélection plus rapide vers la productivité qui pourrait compenser les changements de pratiques agricoles. Des observations de caractères agromorphologiques qui pourraient intervenir dans le rendement ont montré d'abord qu'il y a une variabilité importante dans les formes architecturales des plantes et de leurs surfaces foliaires. Comme pour d'autres espèces (Triboi et TriboiBlondel, 2002), les premiers travaux ont démontré l'importance de la période de remplissage du grain pour le tournesol. Debaeke et al. (2004) et Triboi-Blondel et al. (2004) ont mis en évidence le rôle du maintien de l'activité photosynthétique en postfloraison, déterminé par le maintien de la surface foliaire active et l'activité métabolique de cette surface. Le gain de rendement d'environ $50 \%$ entre les variétés cultivées dans les années 1960 et celles culti-

\begin{abstract}
In sunflower, the active leaf area during the grain filling period has been shown to be an important character, which can be used to predict seed yield. Total and residual leaf areas of inbred lines and their hybrids are significantly correlated, but there may be some interaction between parents. Strict sense heritability is moderate. The relation between residual leaf area and seed yield has been confirmed using simplified leaf measurements on recombinant inbred lines and estimation of leaf area index with a ceptometer on hybrids. Studies of use of residual leaf area as a character in breeding for seed yield showed that F2 or F3 plants with the smallest leaf areas can be discarded, but, according to genotype, those with medium or largest leaf area give the best seed yields.
\end{abstract}

Key words: flowering, hybrid, inbred line, inheritance, leaf area, prediction vées en 2000 (Vear et al., 2003), de 30 à $45 \mathrm{q} / \mathrm{ha}$ dans un réseau d'essais en 2001 et en 2002, a été accompagné, et peut-être obtenu, par une augmentation de l'indice foliaire total (surface foliaire par mètre carré de sol) de 2,5 à 6,5 en Limagne. Il a été démontré que le trait morphologique le mieux corrélé au rendement était la surface foliaire active trois à quatre semaines après la floraison. Cette surface est en partie déterminée par la surface foliaire totale (SFT) à la floraison, dont la corrélation avec le rendement est significative mais plus ou moins étroite selon les conditions de culture. Triboi-Blondel et al. (2004) et Lecoeur et al. (2010) distinguent deux types d'architecture pour le tournesol: le type «sapin», avec les plus grandes feuilles positionnées assez bas, et donc peu ombragées par les feuilles supérieures, et le type «pin parasol», avec les plus grandes feuilles situées très haut sur la plante. Généralement, les formes "pins parasols » correspondent à des variétés assez courtes, tandis que les formes « sapins » sont associées à des variétés plus hautes. Parmi les variétés récentes, les formes «sapins » prédominent, mais aucune relation étroite avec le rendement n'est apparue. C'est pourquoi, pour les premières études de génétique, nous avons commencé par les caractères plus simples que sont les surfaces foliaires.
Pour pouvoir utiliser des mesures de surface foliaire en sélection ou en études génétiques à grande échelle, il faut que les mesures soient faciles et répétables et que l'hérédité du caractère soit connue. Les résultats présentés ici concernent les relations entre les surfaces foliaires de lignées et leurs hybrides, mesurés pendant quatre ans, ainsi que quelques expérimentations de l'utilisation de mesures simplifiées, en sélection ou en essai pour de grands nombres de parcelles.

\section{Analyses de l'héritabilité}

\section{Matériel}

Vingt-quatre hybrides d'un plan de croisement factoriel partiel entre cinq lignées femelles $(F)$ et huit lignées restauratrices $(M)$, ainsi que ces lignées parentales, ont été conduits en essais de deux blocs randomisés, en deux lieux (Clermont-Ferrand et Toulouse) en 2003 et en 2004. En 2004, le croisement factoriel complet, $5 \mathrm{~F} \times 8 \mathrm{M}$, soit 40 hybrides, a été implanté à Clermont-Ferrand.

\section{Méthodes}

La SFT a été déterminée par des mesures à deux dates, un premier passage avant floraison pour les feuilles les plus âgées ( 1 à 15 par exemple) 

second passage après floraison quand toutes les feuilles ont terminé leur croissance. Pour chaque feuille, la longueur et la largeur ont formule $L \times \mid \times 0,713$.

La surface foliaire résiduelle (SFR) a été déterminée pour chaque plante, une fois par semaine, à partir de la floraison, par le suivi du jaunissement de chaque feuille et le calcul de la perte de surface verte (active) par rapport à la surface totale.

\section{Résultats}

Les valeurs moyennes des lignées et des hybrides sont présentées dans le tableau 1 . Les lignées femelles se classent exactement de la même façon pour SFR que pour SFT (dans l'ordre décroissant: VHQ, XRQ, VAQ, FNRM, $V D Q)$, les vitesses de dessèchement étant semblables, sauf en ce qui concerne VDQ, qui est plus précoce que les autres et dont la valeur de SFR décroche par rapport à SFT. Les lignées mâles montrent une plus grande variabilité que les femelles pour SFT et SFR, la lignée PAR6 ayant une surface foliaire plus de deux fois supérieure à PPR9 et à PST3. Comme pour les femelles, les valeurs de SFR se classent à peu près dans le même ordre que les valeurs de SFT. avant que leur dessèchement ne débute, un été mesurées. La surface est calculée par la

Les valeurs des hybrides montrent bien l'hétérosis pour la surface foliaire qui est, en général, le double de celles des lignées. Les corrélations entre les moyennes des deux lignées parentales et de leurs hybrides sont élevées : $r=0,73$ pour SFT et 0,79 pour SFR (figure 1). Ainsi, puisque les mesures sur les parents expliquent $60 \%$ de la variabilité entre hybrides, il serait possible de prédire assez correctement la morphologie des hybrides à partir de mesures sur des lignées. Néanmoins, I'hérédité n'est pas entièrement additive. Une analyse factorielle sur l'essai 2004, à Clermont-Ferrand, a montré des effets femelles et mâles hautement significatifs mais aussi des interactions femelles-mâles significatives pour SFT et pour SFR (tableau 2).

\section{Possibles applications en sélection}

\section{Mesures simplifiées sur un grand nombre de lignées en comparaison avec les rendements de leurs hybrides}

\section{Matériels et méthodes}

Pour 280 lignées recombinantes (RIL) issues de I'hybride Inedi, les valeurs de SFT et de SFR ainsi que les poids de semences obtenues en fécondation libre (le potentiel) furent mesurés. Pour

Tableau 1. Surfaces foliaires totales (SFT) et résiduelles (SFR) moyennes de lignées de tournesol et de leurs hybrides à Clermont-Ferrand et à Toulouse en 2003 et en $2004\left(\mathrm{en} \mathrm{cm}^{2}\right)$.

\begin{tabular}{|llllll|}
\hline Lignées & SFT & SFR & Hybrides & SFT & SFR \\
\hline Femelles & & & XRQ PAR6 & 7700 & 6755 \\
XRQFNRM & 6040 & 3918 & XRQ PSC8RM & 7851 & 4567 \\
FNRM & 3829 & 2593 & XRQ PST5RM & 5216 & 5971 \\
VAQ & 5429 & 3579 & XRQ OPB4 & 10263 & 5997 \\
VHQ & 6521 & 4250 & XRQ PPR9 & 6795 & 3819 \\
VDQ & 3365 & 1928 & FNRM PAR6 & 8387 & 5061 \\
& & & FNRM PSC8RM & 6146 & 3580 \\
Mâles & & & FNRM PST5RM & 4521 & 4115 \\
PAR6 & 7107 & 3819 & FNRM PPR9 & 5860 & 3011 \\
PSC8RM & 4018 & 1682 & FNRM QPR6 & 6843 & 5114 \\
PST5RM & 3231 & 1554 & VAQ PAR6 & 11227 & 6931 \\
OPB4 & 5244 & 2717 & VAQ PSC8RM & 7691 & 5448 \\
PPR9 & 2950 & 1496 & VAQ PST5RM & 4212 & 4485 \\
QPR6 & 3314 & 2101 & VAQ OPB4 & 7599 & 4877 \\
PST3 & 2190 & 635 & VAQ PPR9 & 6459 & 4358 \\
& & & VHQ PSC8RM & 7574 & 4634 \\
& & & VHQ PST5RM & 4714 & 4374 \\
& & & VHQ QPR6 & 7981 & 5932 \\
& & & VHQ PST3 & 4981 & 4546 \\
& & & VDQ PSC8RM & 6106 & 4535 \\
& & VDQ PST5RM & 4128 & 3974 \\
& & & VDQ OPB4 & 6631 & 4158 \\
& & & VDQ PPR9 & 6102 & 3789 \\
& & & 4338 & 3724 \\
\hline
\end{tabular}

120 d'entre elles, on disposait déjà des rendements moyens de sept essais des hybrides entre ces lignées et une même lignée testeur. Des études ayant montré en 2003, d'une part, que les feuilles dont les surfaces sont les mieux corrélées au rendement sont celles numérotées 6 à 15 à partir du capitule et, d'autre part, qu'il est possible d'estimer la surface d'une feuille $n$ à partir des feuilles de rang $n-1$ et $n+1$, il a été décidé de mesurer seulement les feuilles $6,8,10,12$ et 14 .

\section{Résultats}

Les graphiques de la figure 2 montrent des corrélations hautement significatives entre les valeurs de SFT et de SFR et de la production de semences pour l'ensemble des 280 lignées, mais le pourcentage d'explication du rendement est seulement de 20 à $30 \%$. La figure 3 montre les relations entre les surfaces foliaires de 120 de ces lignées et les rendements moyens de leurs hybrides. Les corrélations sont toujours significatives. Si une sélection de la meilleure moitié des lignées pour les SFR avait été réalisée, les lignées donnant les meilleurs hybrides auraient été retenues. La simplification des mesures peut donc être validée.

\section{Choix des meilleures familles \\ pour le rendement en sélection généalogique sur familles F2/F3}

\section{Matériels et méthodes}

Les descendances issues de croisements entre des lignées contrastées pour leurs surfaces foliaires, femelles XRQ et FNRM et mâles PAR6 et PST5RM, ont été suivies. Les SFR de 100 plantes F2 ont été mesurées en 2003, et les 25 meilleures et 25 moins bonnes retenues. Leurs descendances F3 ont été caractérisées en 2004 et des hybrides fabriqués par croisement avec une lignée testeur (VHQ pour PAR6 × PST5RM et PSC8gms pour XRQ $\times$ FNRM). Ces hybrides ont été expérimentés en 2005 et en 2006.

\section{Résultats}

Les corrélations entre les SFR des plantes F2 et des moyennes de leurs familles F3 sont significatives pour les deux séries: PAR6 $\times$ PST5RM : $r=0,440 * *$ XRQ $\times$ FNRM : $r=0,307^{*}$. Les relations entre les SFR et les rendements en grains des hybrides présentent des résultats différents selon le croisement. Pour PAR6 × PST5RM (figure 4A), la corrélation SFRrendement est hautement significative $\left(r=0,49^{* *}\right)$. Une sélection pour les familles F3 avec les meilleures SFR aurait permis de garder celles ayant les meilleures aptitudes à la combinaison. En revanche, la meilleure régression entre rendement et SFR pour XRQ $\times$ FNRM était de deuxième degré (figure $4 B$ ), faisant conclure que, si on pouvait bien éliminer les familles à très faible SFR, celles avec les plus 

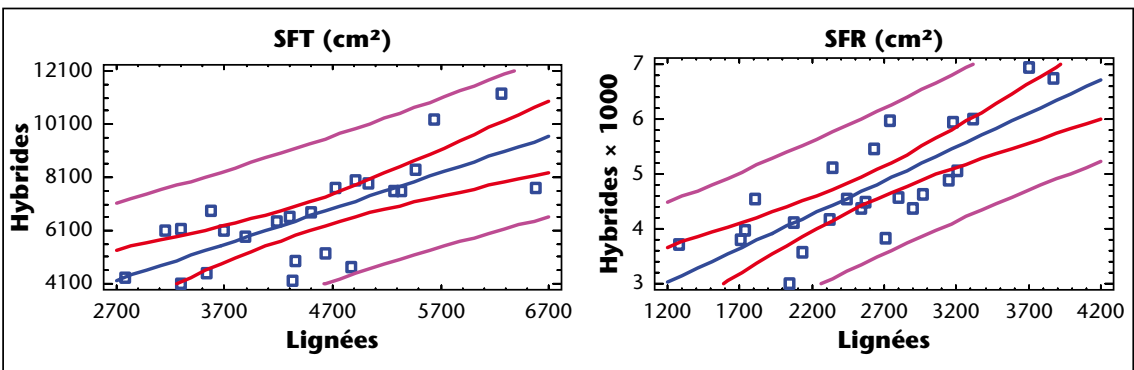

Figure 1. Régressions des surfaces foliaires totales (SFT) et résiduelles (SFR) d'hybrides de tournesol sur les moyennes de leurs lignées parentales.

Tableau 2. Effets parentaux et interactions dans une analyse factorielle sur les surfaces foliaires totales et résiduelles

\begin{tabular}{|lllll|}
\hline & $\mathbf{F}_{\text {femelles }}$ & $\mathbf{F}_{\text {mâles }}$ & $\mathbf{F}_{\text {Interactions }}$ & $\mathbf{h}^{\mathbf{2}}$ \\
\hline SFT & $15,29^{* *}$ & $32,32^{* *}$ & $9,44^{* *}$ & 0,63 \\
\hline SFR & $8,21^{* *}$ & $22,97^{* *}$ & $6,71^{* *}$ & 0,51 \\
\hline
\end{tabular}
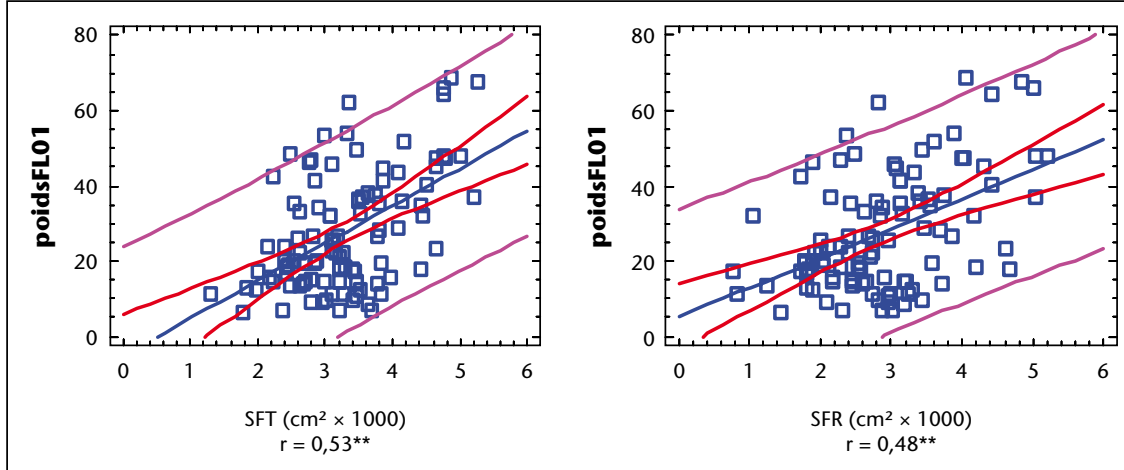

Figure 2. Régression des productions grainières en fécondation libre (poids FL01) de lignées recombinantes de tournesol sur leurs surfaces foliaires totales (SFT) et résiduelles (SFR).
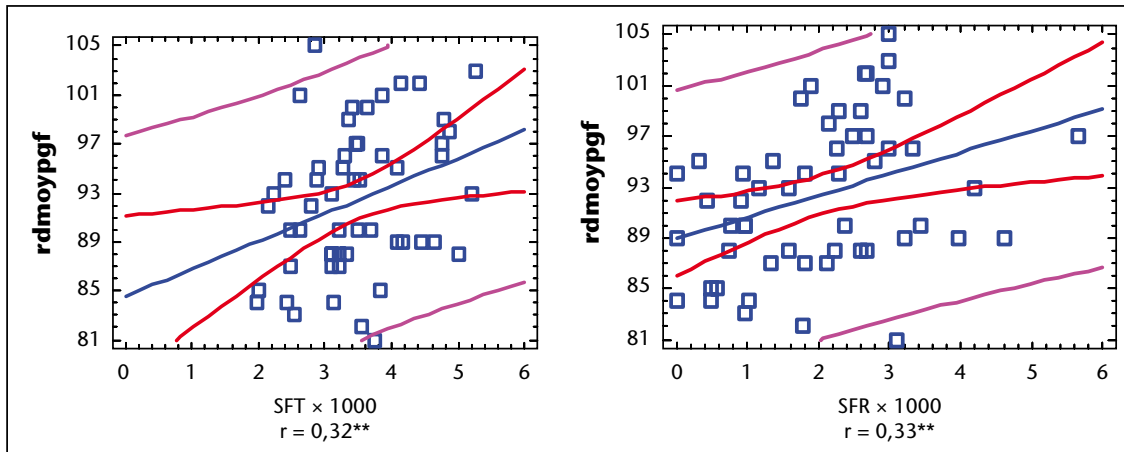

Figure 3. Régressions des rendements en grains d'hybrides de tournesol (rdmoypgf) sur les surfaces foliaires totales (SFT) et résiduelles (SFR) de leurs lignées recombinantes parentales.

grandes surfaces (plus de $3000 \mathrm{~cm} /$ plante) ne donnaient pas de bons rendements non plus. La gamme des surfaces foliaires des descendances des deux croisements étant semblable, de 1000 à $5000 \mathrm{~cm}^{2}$ par plante, il faut suggérer que la différence vient d'une différence dans l'efficacité de transfert des feuilles et la tige aux graines (l'indice de récolte).
Utilisation d'un ceptomètre pour mesurer les indices foliaires, sur les hybrides avec une collection de lignées diverses

\section{Matériels et méthodes}

Des hybrides entre quatre testeurs et une core collection de 144 lignées (Coque et al., 2008) ont été expérimentés à Clermont-Ferrand en 2008, dans un ensemble de 480 parcelles (huit essais de 30 variétés et deux répétitions avec trois variétés témoins dans chaque essai). Les tailles des plantes et les rendements en grains ainsi que les surfaces foliaires ont été mesurés. Pour cela, un ceptomètre a été employé; ce capteur portable compare le rayonnement au-dessus du couvert à la radiation transmise à la base des feuilles actives dans le couvert, ce qui permet une estimation directe et non destructive de l'indice foliaire de la parcelle. Les mesures de rayonnement ont été réalisées à la floraison (SFT) et 30 jours plus tard (SFR). Cette méthode a l'avantage de prendre moins d'une minute par parcelle, pour obtenir la moyenne de cinq mesures au lieu de dix minutes environ pour les mesures classiques décrites plus haut.

\section{Résultats}

La figure 5 montre des corrélations hautement significatives entre les rendements en grains et les indices foliaires, plus particulièrement pour les mesures réalisées 30 jours après la floraison, SFR expliquant $38 \%$ de la variance du rendement $\left(r=0,62^{* *}\right)$. II semble donc qu'on peut valider ce système de mesure rapide pour avoir une prévision, ou une explication partielle, des rendements en grains. Cependant, une comparaison des tailles de plantes avec les rendements en grains montre aussi une corrélation significative $\left(r=0,67 ; \mathrm{R}^{2}: 26 \%\right)$. Une régression multiple, impliquant SFR et la taille, a donc été calculée ( $\mathrm{rd}=0,448$ lai208 (=SFR) $+0,651$ taille $-21,27$ ). Elle explique $47 \%$ de la variabilité des rendements parmi les hybrides en essai.

\section{Discussion}

Dans la littérature, il y a peu d'études de l'hérédité de la SFT ou de la SFR du tournesol. Cela résulte, d'une part, des efforts prioritaires sur les caractères dont l'importance pour la sélection est directement évidente (rendement en grains, teneur en huile, taille, précocité de floraison et de maturité, etc.) et, d'autre part, du coût assez important pour faire les mesures de surfaces foliaires sur un nombre suffisant de plantes. Puisqu'il a été démontré ici que la surface foliaire active trois à quatre semaines après la floraison ainsi que la SFT sont de bons prédicteurs du rendement, la connaissance de 




Figure 4. Régressions des rendements en grains d'hybrides de tournesol ( $r d 2005$ et rd2005vh) sur les surfaces foliaires résiduelles (SFR) moyennes des familles F3 obtenues à partir de deux croisements : a) deux lignées restauratrices, b) deux lignées « femelles».



Figure 5. Régressions des rendements en grains d'hybrides de tournesol (rdraptem) sur leurs indices foliaires à la floraison (laifloraptem) et résiduels 30 jours après la floraison (lai208raptem). Les rendements et les indices foliaires sont exprimés en rapport aux témoins des essais.

la génétique de ces caractères devient importante pour améliorer l'efficacité de la sélection pour le rendement en grains.

L'héritabilité de SFR et de SFT au sens strict est de niveau moyen, comme l'avait trouvé Simon (1999) pour la surface foliaire de la dernière feuille chez le blé et Oram et al. (1974) pour la teneur en azote des feuilles de Phalaris tuberosa (une culture fourragère). Néanmoins, même $s^{\prime}$ il y a des interactions entre lignées parentales dans un hybride, des connaissances sur les surfaces foliaires des parents permettent de prédire assez bien celles des hybrides.

La relation SFR-rendement a été mise en évidence sur une large gamme de génotypes de tournesol cultivé, lignée ou hybride, et doit donc pouvoir être exploitée. La possibilité de réaliser des mesures rapides d'indices foliaires avec un ceptomètre ou un capteur optique devrait permettre d'augmenter le nombre de mesures afin d'approfondir les connaissances en génétiques et d'approcher la génomique. sont une illustration simple. Le modèle développé par Casadebaig (2008), Lecoeur et al. (2010) et Debaeke et al. (2010), dans ce dossier, a l'objectif d'intégrer au mieux les connaissances éprouvées pour une meilleure prédiction et un diagnostic du rendement. Pour améliorer les connaissances en génétique et en génomique, il va falloir, en plus, inventer d'autres méthodologies de phénotypage efficaces pour mesurer et caractériser les plantes pour des caractères complémentaires de la surface foliaire dans la mise en place du rendement, tels que l'efficience photosynthétique ou l'indice de récolte.

Remerciements. Ces études furent réalisées avec le soutien de PROMOSOL et d'un programme ANR-génoplante «Sunyfuel ». Nous remercions Joelle Messaoud, Sylvie Roche, les équipes des domaines INRA à ClermontFerrand et à Toulouse ainsi que I'URD Agri-obtentions à Clermont-Ferrand pour leur aide dans la réalisation des essais.

\section{RÉFÉRENCES}

Casadebaig P. Analyse et modélisation des interactions génotype-environnement-conduite de culture : application au tournesol (Helianthus annuus). Doctoral thesis INP Toulouse, France, 2008.

Coque M, Mesnildrey S, Romestant M, et al. Sunflower line core collections for association studies and phenomics. Proc. 17th Int Sunflower Conf, Cordoba (Spain), 2008, 725-8.

Debaeke P, Casadebaig P, Haquin B, Mestries E, Palleau JP, Salvi F. Simulation de la réponse variétale du tournesol à l'environnement à l'aide du modèle SUNFLO. OCL $2010 ; 17$ : 143-51.

Lecoeur J, Poiré-Lassus R, Christophe A, et al. SUNFLO: a joint phenotyping and modelling approach to analyse and predict differences in yield potential of sunflower genotypes. Funct Plant Biol 2010 (submitted).

Oram RN, Clements RJ, McWilliam JR. Inheritance of nutritive quality of summer herbage in Phalaris tuberosa L. Aust J Agr Res $1974 ;$; 25 : 265-74.

Simon MR. Inheritance of flag-leaf angle, flag-leaf area and flag-leaf duration in four wheat crosses. Theor Appl Genet 1999 ; 98 : 310-4.

Triboi-Blondel AM, Messaoud ], Debaeke P, Lecoeur ], Vear F. Heredity of sunflower leaf characters useable as yield predictors. Proc 16 th Int Sunflower Conf, Fargo (USA) $2004 ; 2: 517-24$

Triboi E, Triboi-Blondel AM. Productivity and grain or seed composition: a new approach to an old problem. Eur J Agron 2002 ; 16 : 163-86.

Vear F, Bony $H_{\text {, Joubert }} \mathrm{G}$, Tourvieille De Labrouhe $\mathrm{D}$, Pauchet I, Pinochet $X .30$ years of sunflower breeding in France. OCL $2003 ; 10: 66-73$. 